\title{
UNIVERSAL SYSTEM IN BUSINESS
}

\section{PRAFULLA K SWAIN \& BIBHUTI B PRADHAN}

Department of Management, Siksha 'O' Anusandhan (Deemed to be University), Bhubaneswar, Odisha, India

\begin{abstract}
Robot programming can be a tedious task, particularly in industrial environments where different types of robots are required, and in flexible automation lines where robots often need to be reconfigured. In this respect there are some shortcomings to be overcome. Time-efficiency is the main problem. This paper proposes a new robot programming solution, a mobile application called Uii Mobile, intended to bring the smartphone to the shop floor while enabling robot programming for operators with limited technical knowledge. Using the intuitive graphical user interface (GUI) for Uii Mobile eliminates the time required by a worker to learn various programming languages.Uii Mobile also allows for faster reconfiguration of robots for small, versatile tasks like picking and placement. Instead, non-standard interfaces, like robots, are used to control machinery; The problem is, firstly, that operators need training to use this equipment, as most robots ' design is not intuitive. Nonetheless, a user analysis using the System Usability Scale (SUS) scores Uii Mobile with a score of 75.38/100 which makes it an above-average usability system.

KEYWORDS: Industry 4.0, IoT, Smartphones, Industrial Robots, Flexible Automation, Human-Machine Interaction, Usability
\end{abstract}

Received: Jun 08, 2020; Accepted: Jun 28, 2020; Published: Aug 26, 2020; Paper Id.: IJMPERDJUN2020900

\section{INTRODUCTION}

Smartphones are used throughout the globe by people of nearly all ages and for many it has become the primary technological platform for accessing information, entertainment and communication. Smartphones have become an integral part of our everyday, social life, and they have an easily extendable, familiar interface with millions of available applications. Recently, smartphones have become interfaces for controlling or at least tracking specific devices (e.g. wireless speakers, vacuum cleaners, etc.) by linking to the Internet of Things (IoT). Smartphones, however, become redundant once they move into a factory with automated production lines. Instead, non-standard interfaces, like robots, are used to control machinery; The problem is, firstly, that operators need training to use this equipment, as most robots ' design is not intuitive. Second, almost all of these controllers have their own programming language, so when moving from one robot to another, both language so interface need to be changed. In response to this question, we are proposing a quick, intuitive teach-pend-replacement of an Android smartphone running.

The program is cross-vendor compliant in the spirit of Industry 4.0, and is applicable to any robot setup. Uii's interface is simple and intuitive, greatly reducing the need for comprehensive training before programming robots; thus allowing robotics novices to program robots. The layout of this paper is as follows: Section 2 discusses similar field study, followed by Section 3 that introduces the Uii Platform including work on Uii Desktop, a computer device, as well as Uii Mobile, the above-mentioned Android application. 


\section{LITERATURE WORK}

As seen through the emergence of Industry 4.0, Smart Manufacturing and similar initiatives, the need for more agile manufacturing solutions is imminent. This is not least true within the field of industrial robotics, where robots will have to take on smaller and smaller batch sizes with ever increasing variance. As a result, more intuitive and faster means of reconfiguring robots is needed as opposed to traditional robot programming using non-standard teach pendants that are different across devices from different producers. Furthermore, since most industrial robots today feature proprietary interfaces and programming languages, it becomes challenging for operators to learn and operate robots of various brands[1]-[4].

One solution explored by several researchers is to create an intuitive robot programming tool using the familiarity of a smart phone. Not only is the modality of interacting through the touch screen is familiar to most, but the smart phone also provides a suite of additional sensors. Both Lambrect et al. and Yepes et al. utilize the accelerometer of a smartphone to create an intuitive way of jogging the robot end-effector by tilting and turning the phone itself. Yepes et al. conducted a small usability study in which $72 \%$ of the participants found a smart phone an adequate tool for controlling a robot.

In extend of controlling the end-effector, a smart phone application must be able to store and execute tasks to successfully replace existing teach pendants. Jan et al. presents the Android application called "Smart teaching pendant" which offers both a teaching mode and an operation mode. In operation mode the "Smart teaching pendant" allows the operator to play, pause and skip the current task. The tool is only tested against a simulated robot, which is perhaps why no information on how the programming is conducted is included.

$\mathrm{Su}$ and Young presents a tablet-based interface offering both robot control and task programming and execution. The interface is tailored for a 6-DOF robot from Industrial Technology Research Institute in Taiwan, but it works both online with the real robot and offline with a simulated robot. As in, very little focus is put on describing how the programming and subsequent execution of tasks is done.

Mateo et al. presents "Hammer"; a comprehensive robot interface implemented in Android and running on a tablet. The interface features both robot control, task programming and task execution. Furthermore, it includes a graphical drag-and-drop programming IDE and augmented reality. In, "Hammer" is applied together with a COMAU robot but intended for use with any robot brand. However, the paper does not disclose details on the implementation or the interface towards the hardware.

Lambrecht et al. introduce a standardized interface between their smart phone application and the specific hardware. It consists of a generic robot language with a set of generic functions used by the application[5], [6]. When invoked, these generic functions are then translated into the language of the specific robot controller by an interpreter. Similar to the work presented in we propose a smart phone application serving a as a replacement for the teach pendant when programming and operating industrial robots. Contrary to the work presented above, we emphasize the system level architecture and the use cases of both programming and executing tasks on an industrial robot. Our framework is independent of robot vendor, which we demonstrate with two robots; a KUKA and a Universal Robots. Lastly, we conduct a study with participants having varying robotics knowledge to explicitly assess the usability of the smart phone application. 


\section{UNIVERSAL INDUSTRIAL INTERFACE FRAMEWORK}

The smartphone app itself is only one part of the solution that enables industrial robot control through a smartphone. The overall framework, called Uii framework, consists of two primary pieces, as illustrated on Fig. 1. Uii Desktop is the core of the system and provides the communication towards the equipment (robot, gripper, etc.) along with the programming and execution engines. Uii Desktop is intended to run on a PC or Raspberry Pi with a wired connection to the equipment over e.g. LAN. Uii Mobile runs on an Android smartphone, and serves as the front-end towards the user. Uii Mobile and Uii Desktop communicate via a TCP/IP connection.

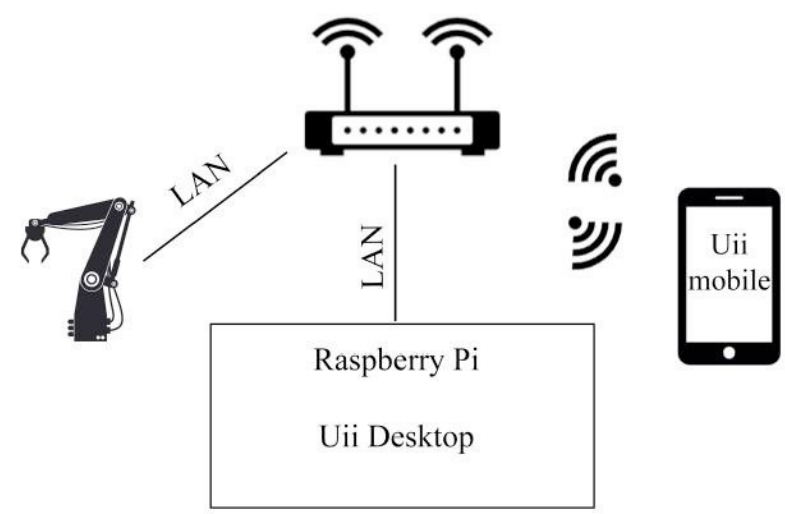

Figure 1: Uii Framework Setup.

\section{Uii Desktop}

Uii Desktop is a vendor independent, cross-platform software meant to facilitate robot programming by introducing a GUI based programming approach rather than code. Uii Desktop was developed previously in, and it in itself also provides a front-end user interface, through which robot programming and task execution can be done. However, in this paper we propose an extension of Uii Desktop, allowing a remote front end to be used from a smartphone (Uii Mobile). Uii Desktop as well as the desired manipulators and grippers must be connected to the same local area network (LAN) in order to establish a TCP/IP connection which is necessary for communication and data transfer. In this case the robot's controller hosts the server sockets which receives requests from the client sockets located on the computer running Uii Desktop. The low-level communication between the hardware is done through specific drivers. Fig. 2 is a representation of how specific hardware is integrated with Uii Desktop[7]-[9].

The user interface of Uii Desktop is split into tabs for different functionalities. The fist tab is the welcome tab, which is meant for establishing connection to a robot. A robot configuration has a name and it is composed of one manipulator and one gripper. The user can create a new robot configuration in the welcome tab by providing a name for the robot configuration, by selecting what type of manipulator the configuration has (Kuka, UR5 etc.) and what type of gripper the robot has (Schunk, Robotiq, pneumatic etc.), and by inserting the IP address for each device. After connecting to a specific robot, a new tab is automatically displayed, called the Robot tab, which contains arrow shaped buttons for manipulating orientations and positions of the manipulator, as well as gripper actions. Uii Desktop offers the option of connecting to multiple robots at the same time via a multi-threading method. In this case, the program opens a jogging tab for each connected robot. This jogging interface makes it possible to control a robot from a computer, such as a Raspberry Pi equipped with a touch screen, or a regular personal computer[10]-[12]. 
In extension of Uii Desktop following the development of Uii Mobile, the welcome screen contains another option, meant for remote control. At the click of a button, a server is opened for a robot, which displays a new tab with a QR code containing parameters needed to establish connection to a robot, as well as status lights which serve as connectivity information for the manipulator, gripper and the smartphone. The status lights light up green, yellow andred in case of ready to execute a command, the component is executing a command and fault respectively. This function is meant to give a visual feedback to the user, as the status lights are visible from a distance and an operator can attend in case of need. Fig. 2 shows the user interface of Uii Desktop when being used in remote control mode.

\section{Usability Study}

A user analysis was performed with 26 research users to evaluate the intuitive and user experience while using the Uii platform with the Uii Mobile App. The participants ranged from people with no professional experience to people with robotics expertise. Enrolling people with varying expertise in robotics was deliberate, since the Uii platform is intended not just for novices but also for advanced users. The System Usability Scale (SUS) was used to evaluate the user experience. SUS is a commonly used UX testing device in the systems engineering market.

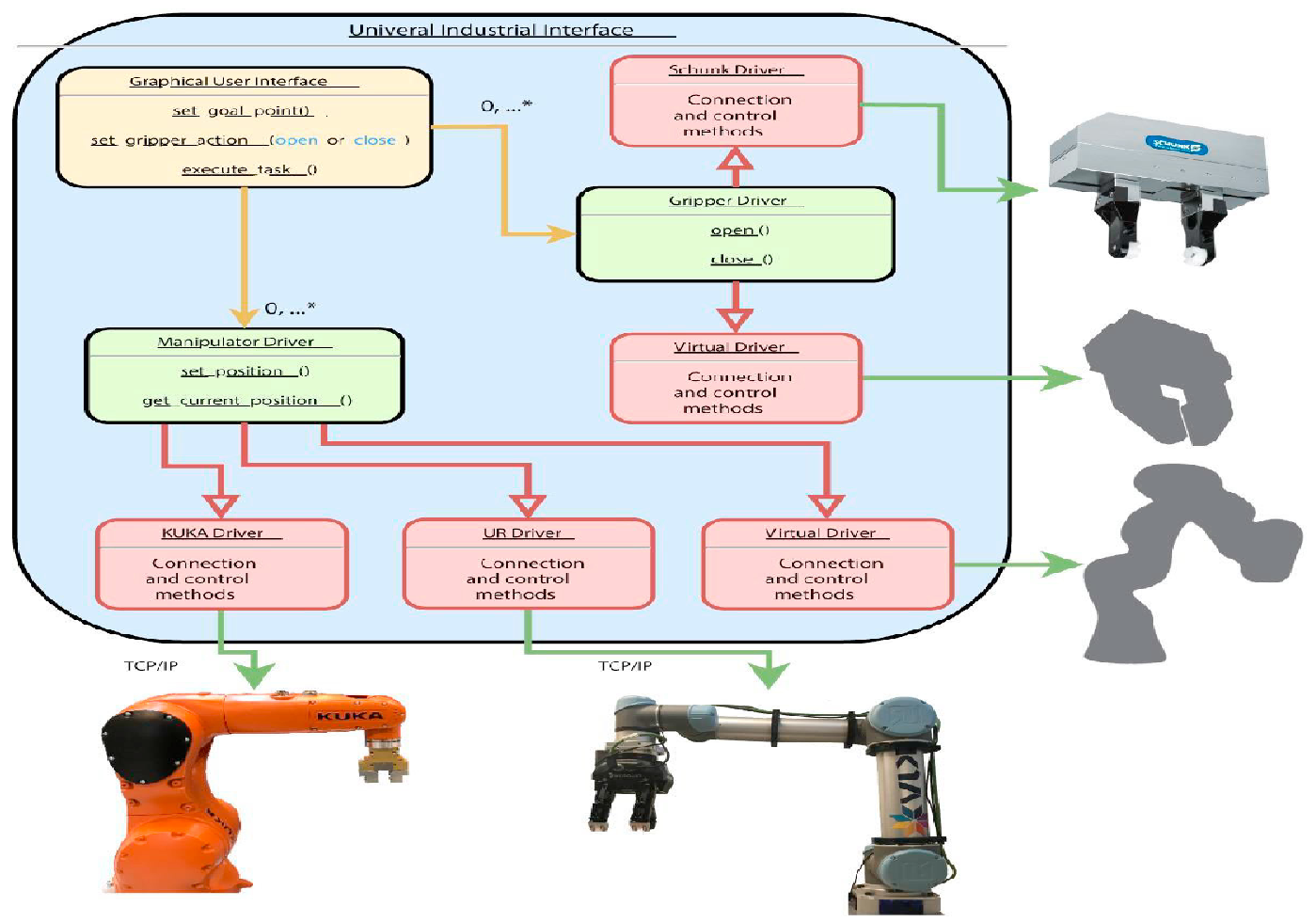

Figure 2: Uii Desktop Architecture.

\section{Protocol}

The participants carried out the test individually, with no interaction between them. The procedure for each of the participants was as follows:

\section{Instruction/Training}

A sheet of instructions has been presented to each participant. This assured that everyone gets the exact same information prior to the test, thus avoiding the contamination of the data. 


\section{Task Programming}

They were asked to program a task using Uii Mobile. In order to do this, the subject had to:

- Start Uii Desktop and connect to a robot and gripper via Uii Desktop.

- Start the Uii Mobile and connect to Uii Desktop.

- $\quad$ Program a simple pick and place task.

- Modify several points in the existing program.

- Execute the programmed task.

\section{Survey}

After conducting the test, the subjects had to fill in the SUS survey. The survey contained 10 questions on the SUS scale, with options from 1 (Strongly agree) to 5 (Strongly disagree). These questions follow the standard SUS format which can be found. To acquire qualitative data as well, the subjects with robotics experience were asked to provide their personal qualitative assessment of Uii Mobile and to propose improvements.

\section{RESULTS}

When analyzing and interpreting the results of the SUS, the overall score can range between 0 and 100 . If the system's score is below 68 it is considered a system with under average usability. And everything above the value of 68 is considered a system with over average usability. Uii Mobile scored an average of 75.38. More about SUS and result interpretation can be found at [13]. The participants found it easy to get acquainted to the system, and the majority considered they would not need the help of a technical person to use this app. Regarding the qualitative data, a major finding, which also complies with the requirements of the system, was that people with experience within the field of robotics found Uii Mobile to be easier to use than traditional teach pendants for fast reconfiguration of robots.

\section{CONCLUSIONS}

As stated in the introduction of this paper, industrial and collaborative manipulators ' programming process can present problems in terms of time and finances. The key reason for this is the lack of a consistent interface across robots irrespective of their manufacturer, as discussed in this paper. Operators often communicate with robots (industrial or collaborative), in a versatile manufacturing environment. By shortening the time taken to reprogram them and providing a familiar operator interface, a new product will enter the market more quickly. Smartphones are apps that everyone is familiar with. A successful attempt was made through the development of Uii Mobile on top of Uii Desktop to incorporate smartphones into the industrial production environment and obtain a consistent interface across robotic manipulators irrespective of their manufacturer. This paper addressed only the use of the system on manipulators for Kuka and Universal Robots, but a library that includes as many tools as possible is the focus of further development of the Uii program as a whole.

Nonetheless, the key aim of providing an open user interface is achieved successfully. There are of course the health issues about this approach when contemplating industrial robot programming. Uii is not intended to replace all of the robotic manipulator's components as they are, but rather to be an adjacent framework that helps to combine multiple devices from different vendors under one interface. Therefore, the operators must be aware of the safety guidelines 
surrounding industrial robots, even whether they are using Uii or not. One feature that can be added on Uii Mobile to enhance the operators ' and the manipulator's own protection is to ensure that the operator is in the robot's visual proximity. This is yet another topic for Uii's further development. Uii can also be further developed as a smartphone application in a viable IoT feature. The data can be transmitted in real time over the internet from the robots to the device, and the robot cell can be tracked remotely at any time.

\section{REFERENCES}

1. P. Corredor and S. Goñi, “TQM and performance: Is the relationship so obvious?,” J. Bus. Res., 2011.

2. M. J. Chappell and L. A. LaValle, "Food security and biodiversity: Can we have both? An agroecological analysis," Agric. Human Values, 2011.

3. P. Anu, "Integrated Public Service Delivery in Agile e-Government System through Service Oriented Enterprise Model in the Context of India,” Electron. Gov. Electron. Particip. Jt. Proc. Ongoing Res. Proj. IFIP WG 8.5 EGOV ePart 2015, 2015.

4. O. Kupervasser, H. Nikolic,, and V. Zlatic, “The Universal Arrow of Time," Found. Phys., 2012.

5. G. Atti, “Lean management," in Quality Management: Tools, Methods and Standards, 2019.

6. P. J. Martínez-Jurado and J. Moyano-Fuentes, "Lean management, supply chain management and sustainability: A literature review,” J. Clean. Prod., 2014.

7. A. Sanders, C. Elangeswaran, and J. Wulfsberg, "Industry 4.0 implies lean manufacturing: Research activities in industry 4.0 function as enablers for lean manufacturing," J. Ind. Eng. Manag., 2016.

8. G. Ballard, "Lean construction," in The Routledge Companion to Lean Management, 2016.

9. M. P. J. Pepper and T. A. Spedding, “The evolution of lean Six Sigma," International Journal of Quality and Reliability Management. 2010.

10. J. S. Toussaint and L. L. Berry, "The promise of lean in health care,” Mayo Clinic Proceedings. 2013.

11. J. Kadarova and M. Demecko, "New Approaches in Lean Management," Procedia Econ. Financ., 2016.

12. S. Hajmohammad, S. Vachon, R. D. Klassen, and I. Gavronski, "Reprint of Lean management and supply management: Their role in green practices and performance," J. Clean. Prod., 2013. 